\title{
Zur Infrastruktur- und Arbeitszeiterhebung in der Psychiatrie
}

Daten, medizinische Daten, ökonomische Daten, werden berufspolitisch zu einem immer wichtigeren Instrument, wenn es darum geht, Anliegen der Ärzteschaft zu vertreten. Dies ist ein platter Gemeinplatz, der kein Editorial verdienen würde.

Daten sind aber auch ein nüchternes Kommunikationsmittel, das über das Befinden des anderen informieren kann. In einer Zeit, da sich immer mehr medizinische Spezialitäten herausbilden und verselbständigen, mit einer eigenen, sich oft den anderen gegenüber verschliessenden Arbeitskultur, wird die Kommunikation mit anderen Ärztinnen und Ärzten und ihren Spezialitäten immer schwieriger. Der Kampf um Schnitze aus einem immer kleineren Kuchen steuert hier noch das Seinige bei.

Die Schweizerische Gesellschaft für Psychiatrie und Psychotherapie und die Schweizerische Gesellschaft für Kinder- und Jugendpsychiatrie und Psychotherapie haben es unternommen, mit Daten, die in dieser Ausgabe publiziert werden [1], die ökonomischen und sozialen Gegebenheiten ihres Arbeitsfeldes - leider nur in der sogenannt Freien Praxis - darzustellen. Sie haben damit nicht nur ein Fundament für ihre Positionierung in Tarifverhandlungen gelegt, sondern ein Bild entworfen, das die Ärztinnen und Ärzte in anderen Disziplinen nachdenklich stimmen muss. Das Bild, das wir «anderen» uns vom Arbeitsfeld der Psychiatrie gemacht haben, zerbröselt zu Makulatur: Nichts hat es mehr auf sich mit dem wortlos seinem Patienten auf der Couch (= Praxisinfrastruktur) zuhörenden, protokollierenden Arzt, der bei tiefen Tarifen aber minimalem Praxisaufwand eine durchaus wohlsituierte Existenz fristet. An seine Stelle tritt das Bild eines Kleinunternehmers, der erhebliche Zeit für Arbeiten aufwendet, deren erfolgreiche Durchführung nicht eigentlich eines akademischen Studiums bedürfte, eines Kleinunternehmers, der nach streng betriebswirtschaftlichen Kriterien nicht selten in den Bankrott gerät, vor dem ihn nur die Arbeit eines Ehepartners zum Nulltarif bewahrt.

Dies ist die real existierende Medizin, die die Medien zur Kenntnis nehmen müssten. Doch noch immer gilt, dass sich Abzocken einzelner medial besser verkauft als das Leid einer Masse von Heloten. Haben wir denn während der Barnevik-Saga je von den Existenzbedingungen der im Putzdienst von ABB arbeitenden Frauen und Männern gehört?

Was bleibt? Wohl nur das unermüdliche In-die-Köpfe-Hämmern dieser Botschaften in der Hoffnung, dass intrakraniell einmal etwas hängen bleibt. Und: Der Aufruf an alle Ärzte und Ärztinnen aller Fachdisziplinen, es den Psychiatern gleichzutun.

\section{Dr. med. Hans Heinrich Brunner}

\footnotetext{
1 Lachenmeier H. Infrastruktur- und Arbeitszeiterhebung Psychiatrie oder «Zu kleine Schuhe» Teil II. Schweiz Ärztezeitung 2002;83(25):1305-9.
} 